\title{
Impacto de la sintomatología depresiva en los resultados de un programa de deshabituación tabáquica en el lugar de trabajo
}

\section{Impact of depressive symptoms on outcomes of a workplace smoking-cessation program}

Gemma Nieva; Eugeni Bruguera; Sergi Valero; Miquel CASAS
Servicio de Psiquiatría. Hospital Universitari Vall d'Hebron /

Universidad Autónoma de Barcelona.
Enviar correspondencia a:

Gemma Nieva. Servicio de Psiquiatría. Hospital Universitario

Vall d'Hebron. Depto. de Psiquiatria y Medicina Legal. Universidad

Autónoma de Barcelona. Pg. Vall d'Hebron 119-129, 08035 Barcelona.

Tel. 9348942 94. Fax: 9348945 87. E-mail: gnieva@vhebron.net.

\section{RESUMEN}

Introducción: Si bien existen evidencias de la relación entre fumar y depresión, poca relevancia se ha dado al impacto de la sintomatología depresiva y antecedentes de depresión en los resultados de programas de deshabituación tabáquica desarrollados en los lugares de trabajo. El objetivo del estudio es analizar la influencia de la sintomatología depresiva y antecedentes de trastorno depresivo mayor (TDM) sobre la abstinencia en el contexto de una intervención laboral y cual es el rol del sexo, dependencia y toma de antidepresivos. Métodos: Estudio retrospectivo cuyos participantes fueron trabajadores del Ayuntamiento de Barcelona que se inscribieron voluntariamente. La intervención consistía en un abordaje multicomponente combinado con tratamiento farmacológico. Para evaluar la presencia de sintomatología depresiva inicial se utilizó la puntuación en el Inventario de Depresión de Beck (BDI). La presencia de antecedentes de TDM se registró en la evaluación basal. Resultados: puntuaciones mayores en $\mathrm{BDI}$ se relacionaron con menor porcentaje de abstinencia a los 3, 6 y 12 meses. Puntuar en $B D I \geq 13$, se asoció a un aumento de la probabilidad de recaída en 1,81 veces. Dicha relación era independiente del efecto de la severidad de la dependencia, del sexo y del tratamiento con antidepresivos. Contrariamente, la presencia de antecedentes no determinó la evolución clínica. Conclusiones: Son necesarias nuevas estrategias terapéuticas especificas para reducir el impacto de la sintomatología depresiva en los programas para dejar de fumar en el lugar de trabajo.

Palabras clave: Dependencia nicotínica, fumar, tabaco, depresión, tratamiento, medio laboral.

\section{ABSTRACT}

Introduction: Although smoking and depression have been related, little importance is given to the impact of depression on outcomes of smoking cessation interventions in the workplace. The aim of this study was to assess the influence of depressive symptoms and a history of depression on abstinence after a workplace smoking cessation intervention, and to explore the roles of gender, nicotine dependence and antidepressants. Methods: A retrospective study with employees of Barcelona City Council participating on a voluntary basis. The intervention consisted of a multicomponent approach combined with pharmacological treatment. Depressive symptoms were assessed with the Beck Depression Inventory (BDI), and history of depression was noted in the baseline assessment. Results: Higher scores in BDI were associated with lower rates of abstinence at 3,6 and 12 months. BDI scores $\geq 13$ were related to a $1.81 x$ higher likelihood of relapse. This relationship was independent of the effect of dependence severity, of gender and of treatment with antidepressants. In contrast, a history of depression did not influence outcomes. Conclusions: New therapeutic strategies are needed to reduce the impact of employees' depressive symptoms in workplace smoking cessation programmes.

Key words: Nicotine dependence, smoking, tobacco, depression, treatment, workplace. 


\section{INTRODUCCION}

E tabaquismo es la causa aislada más importante de morbilidad y mortalidad prematura prevenible en España. En la actualidad un 29,5\% de los españoles mayores de 15 años fuman'. Teniendo en cuenta que la mayoría de la población activa destina la mitad de sus horas de vigilia al trabajo, numerosas publicaciones han evidenciado la idoneidad del lugar de trabajo como marco para desarrollar programas de cesación tabáquica ${ }^{2,3}$. Si bien han mostrado su eficacia, poca relevancia se ha dado al análisis de barreras que impiden mayores porcentajes de abstinencia como la sintomatología depresiva de los trabajadores. Un estudio epidemiológico de trabajadores estadounidenses relacionó el consumo de tabaco y síntomas depresivos, y sugirió que dicha sintomatología podría empeorar los resultados de programas de cesación tabáquica aplicados en el contexto laboral ${ }^{4}$. En realidad, la relación entre fumar y depresión ha sido bien establecida por varios estudios poblacionales ${ }^{5,6}$. La prevalencia de antecedentes de depresión entre fumadores oscila entre el $22 \%$ y el $61 \%$, comparado con el $17 \%$ de la población general ${ }^{7}$. Algunos estudios afirman que haber padecido alguna vez un trastorno depresivo mayor (TDM) empeora la abstinencia tabáquica ${ }^{8,9}$, particularmente en mujeres ${ }^{10,11}$, debido a una mayor intensidad del síndrome de abstinencia (concretamente tristeza y dificultad de concentración ${ }^{12}$ ). Sin embargo, también existen investigaciones que constatan una ausencia de relación ${ }^{13-17}$. Un meta-análisis sobre la cuestión no encontró relación entre tener antecedentes de TDM y abstinencia tabáquica a corto o largo plazo en el contexto de tratamientos intensivos, y ello era independiente del sexo ${ }^{18}$.

Por otro lado, la evidencia apunta hacia una relación directa entre sintomatología depresiva al inicio del tratamiento de deshabituación tabáquica con una mayor severidad de dependencia y peores resultados a corto plazo ${ }^{19}$. Otros estudios han concretado que dicha sintomatología predice el fracaso terapéutico solamente en fumadores con antecedentes de TDM, mientras la relación sería inexistente en sujetos sin historia previa ${ }^{16}$. Hasta el momento, la medicación antidepresiva (a excepción del bupropion y la nortriptilina), no ha mostrado aumentar significativamente la probabilidad de abstinencia tabáquica ${ }^{20}$.

El objetivo del presente estudio es analizar la influencia de la sintomatología depresiva y antecedentes de TDM, sobre la abstinencia a los 3, 6 y 12 meses tras haber iniciado un programa de deshabituación tabáquica en el contexto de una intervención laboral. Asimismo se explora cual es el rol que tienen las variables sexo, la severidad de la dependencia y la toma de antidepresivos (diferentes de bupropion o nortriptilina). De acuerdo con la literatura, se espera encontrar menores niveles de depresión basal en los trabajadores que permanecen abstinentes comparado con los que sufrieron recaídas. En segundo lugar, suponemos que la presencia de antecedentes de depresión no se relaciona con peores resultados, independientemente del sexo, la severidad de la dependencia y la toma de antidepresivos.

\section{MÉTODO}

\section{Sujetos}

Se trata de un estudio retrospectivo cuyos participantes fueron 280 trabajadores del Ayuntamiento de Barcelona que acudieron voluntariamente a un Programa de Cesación Tabáquica (PCT) entre marzo de 2006 y octubre de 2007, y contestaron los cuestionarios. EI PCT fue realizado por el equipo del Programa de Tabaquismo del Servicio de Psiquiatría del Hospital Universitario Vall d'Hebron de Barcelona. El 53,9\% eran hombres y la edad media era de 45,7 años ( $D E=8$; Rango 26-64). El 63,6\% de la muestra estaba casado o vivía en pareja. El 37,5\% tenían estudios secundarios y $46,4 \%$ universitarios. Referente a su ocupación, el 35,2\% formaban parte del cuerpo de la policía local (guardia urbana), el $14,6 \%$ eran técnicos de grado medio, el 10,8\% eran auxiliares, el 7,3\% eran técnicos de grado superior, el 6,3\% trabajaba como administrativos o auxiliar administrativos, el $4,2 \%$ eran educadores/maestros y el 3,5\% eran bomberos; el $18,1 \%$ restante tenía otra ocupación.

\section{Procedimiento}

EI PCT constaba de una visita inicial donde se realizaba una evaluación de la historia tabáquica así como los antecedentes médicos y psiquiátricos. La intervención consistía en un abordaje multicomponente combinado con tratamiento farmacológico durante los tres primeros meses. Se realizaron 9 visitas de 60 minutos, siendo la primera antes de la fecha para dejar de fumar y las siguientes semanales durante el primer mes, después al mes y medio, tres, seis y doce meses. La intervención psicoterapéutica podía ser grupal o individual, siguiendo en ambos casos un modelo cognitivo-conductual haciendo especial énfasis en entrenamiento en habilidades de afrontamiento y prevención de recaídas. Los criterios de inclusión en el tratamiento grupal fueron que el paciente aceptara realizar el tratamiento en grupo y estuviera motivado para dejar de fumar. El único criterio de exclusión fue presentar patología psiquiátrica de difícil manejo grupal. El tratamiento farmacológico consistió en Terapia Substitutiva con Nicotina (TSN), parches y/o chicles o comprimidos de nicotina, bupropion (solo o en combinación con TSN) o vareniclina, siguiendo las pautas recomendadas en la literatura especializada ${ }^{21}$. El tratamiento farmacológico se decidió con el paciente teniendo en cuenta su experiencia previa, expectativas, así como las contraindicaciones descritas. El coste del tratamiento incluyendo la medicación fue asumido por la empresa.

\section{Instrumentos}

Para evaluar la presencia de sintomatología depresiva se utilizó la puntuación obtenida en el Inventario de Depresión de Beck (BDI) en versión adaptada ${ }^{22}$, que contestaron los sujetos antes de la primera visita.

Paralelamente, en la primera visita se interrogó a los sujetos por la presencia de antecedentes depresivos que habian requerido tratamiento psiquiátrico o psicológico. En el recuento de antecedentes depresivos se excluyeron los casos de trastorno adaptativo, considerándolo como una entidad diferenciada. 


\section{Variables analizadas}

Se analizó la relación entre las puntuaciones en BDI y la abstinencia (puntual y continuada) a los 3, 6 y 12 meses desde el inicio del programa. Se evaluó si el sexo, la severidad de dependencia medida con el Fagerström Test for Nicotine Dependence (FTND) ${ }^{23}$, y la toma actual de antidepresivos, tenian algún impacto en dicha relación. Secundariamente se analizó la relación entre puntuaciones en BDI y días hasta la primera recaída (TPR). Adicionalmente, se analizó como se relacionaba la presencia de antecedentes de depresión con los resultados en términos de abstinencia.

La abstinencia puntual fue corroborada por una cooximetría $\leq 10$ ppm (Bedfont, Minismokerlyzer) en las visitas ${ }^{24}$. La abstinencia continuada fue definida como no haber fumado nada (ni caladas) desde el inicio del tratamiento. Se consideró recaída fumar 7 dias seguidos, independientemente de la cantidad ${ }^{25}$. El resto de consumos se consideraron una violación de abstinencia.

\section{Análisis estadístico}

Se incluyeron en el análisis estadístico todos los pacientes de acuerdo con el criterio intention to treat. Se utilizó la prueba de $\bigotimes^{2}$ para las variables categóricas y $t$ de Student para la comparación de medias. Adicionalmente se realizó una análisis de supervivencia siguiendo el modelo de KaplanMeier y el modelo de regresión de Cox, para ello se recodificó la variable BDI en dicotómica considerando presencia de sintomatología depresiva cuando la puntuación era $\geq 13$, tal y como han hecho otros autores ${ }^{26}$. Para estudiar el efecto de las variables sexo y dependencia, sobre la relación entre las puntuaciones en el BDI y la abstinencia se realizó una regresión logística. Posteriormente se analizó el efecto de la toma de antidepresivos. Los valores significativos se aceptaron cuando $p<0,05$. Los análisis se realizaron con el programa SPSS 15.0 para Windows.

\section{RESULTADOS}

\section{Análisis descriptivo}

Los sujetos $(n=280)$ fumaban una media de 21,5 $(D E=10,9)$ cigarrillos al dia y la puntuación media en el FTND era de $5,1(D E=2,6)$. Habían empezado a fumar de forma habitual a los $18,4(D E=3,8)$ años y llevaban $25,3(D E=9)$ años fumando. El 57,9\% habían intentado dejar de fumar al menos una vez.

Un total de $29(10,4 \%)$ fumadores solo realizaron la visita de evaluación y no acudieron a ninguna visita de seguimiento. Iniciaron tratamiento 251 sujetos $(89,6 \%$ del total). Para una descripción del tipo de tratamiento realizado, véase la tabla 1.

La abstinencia puntual $(n=280)$ a los 3 meses fue de $62,1 \%$ y la abstinencia continuada fue del $51,4 \%$. Se excluyó del análisis a un paciente que falleció a los 5 meses desde el inicio del PCT, dejando en el análisis a 279 sujetos. A los 6 y 12 meses, la abstinencia puntual fue del $48 \%$ y $41,9 \%$; y la continuada fue del $41,9 \%$ y $32,6 \%$ respectivamente (figura 1). No se encontraron diferencias significativas en abstinencia puntual o continuada según el tratamiento farmacológico utilizado. El valor medio en TPR $(n=279)$, fue de $184,9(D E=$ 157,1) días.

Tabla 1. Tipo de tratamiento realizado (farmacológico y psicológico).

\begin{tabular}{|c|c|c|c|c|c|c|c|}
\hline & & \multicolumn{5}{|c|}{ TRATAMIENTO FARMACOLÓGICO } & \multirow{2}{*}{ TOTAL } \\
\hline & & NINGUNO & TSN & BUPROPION & TSN + BUPROPION & VARENICLINA & \\
\hline \multirow{2}{*}{ 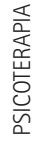 } & Individual, n (\%) & $7(2,8)$ & $11(4,4)$ & $6(2,4)$ & $7(2,8)$ & $7(2,8)$ & $38(15,1)$ \\
\hline & Grupal, n (\%) & $11(4,4)$ & $79(31,5)$ & $40(15,9)$ & $59(23,5)$ & $24(9,6)$ & $213(84,9)$ \\
\hline & TOTAL n (\%) & $18(7,2)$ & $90(35,9)$ & $46(18,3)$ & $66(26,3)$ & $31(12,4)$ & $251(100,0)$ \\
\hline
\end{tabular}

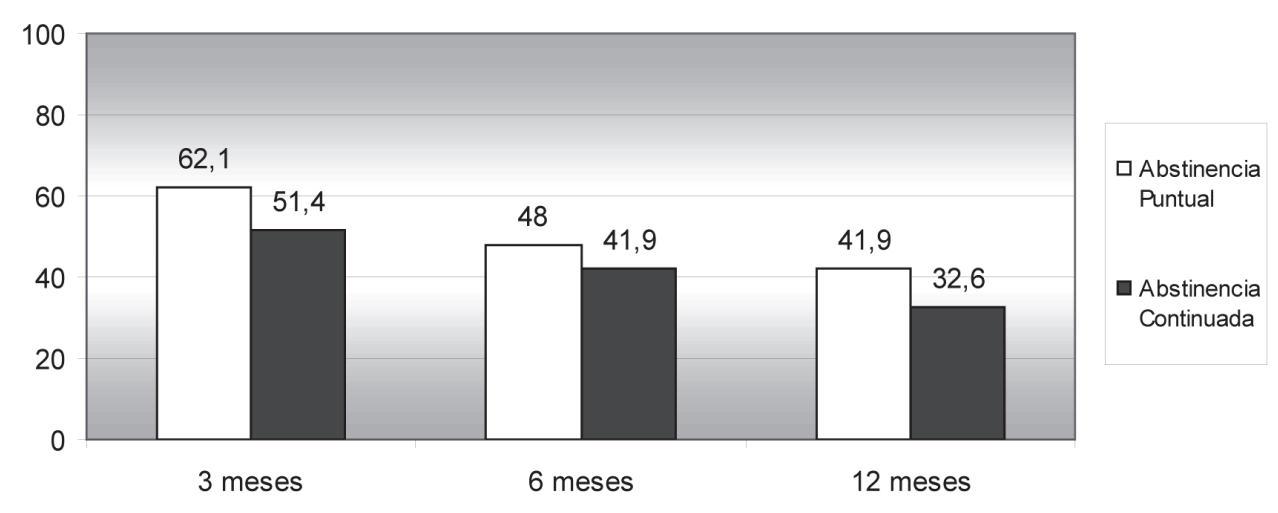

Figura 1. Porcentaje de abstinencia puntual y continuada a los 3,6 y 12 meses $(n=280)$. 
Tabla 2. Puntuación en BDI según abstinencia puntual y continuada a los 3, 6 y 12 meses

(Las diferencias son todas significativas, $\mathrm{p}<0,05$ ).

\begin{tabular}{|l|c|c|c|c|}
\hline \multirow{2}{*}{} & \multicolumn{3}{|c|}{ BD1, media (DE) } \\
\cline { 2 - 5 } & AP1 & No AP & AC2 & No AC \\
\hline 3 meses $(n=280)$ & $7,2(5,4)$ & $9,3(7,7)$ & $6,8(4,9)$ & $9,3(7,5)$ \\
\hline 6 meses $(n=279)$ & $6,8(4,6)$ & $9,1(7,5)$ & $6,4(4,5)$ & $9,2(7,3)$ \\
\hline 12 meses $(n=279)$ & $6,8(4,7)$ & $8,9(7,3)$ & $6,3(4,5)$ & $8,8(7)$ \\
\hline
\end{tabular}

$1 \mathrm{AP}=$ abstinencia puntual

$2 \mathrm{AC}=$ abstinencia continuada

\section{Análisis de la sintomatología depresiva}

La puntuación media en el BDI $(n=280)$ fue de $8(D E=6,4$; Rango 0-36), las mujeres obtuvieron puntuaciones más elevadas $(t=2,35, g l=278, p=0,02)$. No se encontró relación entre las puntuaciones en el BDI e iniciar o no, tratamiento. No se encontraron diferencias significativas entre las puntuaciones de BDI según el tratamiento farmacológico utilizado o el formato de terapia psicológica (grupal o individual).

Sin embargo, tal y como se observa en la tabla 2, puntuaciones mayores en $\mathrm{BDI}$ se relacionaron con menor porcentaje de abstinencia puntual a los 3 meses $(t=2,44, g l=177$, $p=0,016), 6$ meses $(t=3,17, g l=243, p=0,002)$ y 12 meses $(t=2,99, g l=274, p=0,003)$. De manera similar, mayores puntuaciones en $\mathrm{BDI}$ se relacionaron con menor porcentaje de abstinencia continuada a los 3 meses $(t=3,34, g l=278$, $p=0,001), 6$ meses $(t=3,62, g l=277, p<0,001)$ y 12 meses $(t=3,55, g l=255, p<0,001)$. Por otro lado, cada unidad en la puntuación de BDI aumentó el riesgo de recaída a los 3 meses en 1,05 veces (tabla 3 ).

Para la muestra de pacientes que iniciaron tratamiento $(n=251)$, el análisis de supervivencia mostró que los pacientes con sintomatología depresiva (BDI $\geq 13$ ), tardaban de mediana 82 dias hasta la primera recaída, frente a 300 días si $\mathrm{BDI}<13$, diferencia estadisticamente significativa $\left(\nabla^{2}=8,88, g l=1, p=0,003\right.$; figura 2). Puntuar en el BDI 130 más, aumentaba la probabilidad de recaer en 1,81 veces (IC 95\%: 1,22-2,69).

Tabla 3. Regresión logística de la variable sintomatología depresiva (BDI) como predictora de la abstinencia a los 3 meses, sola y ajustado por sexo y severidad de la dependencia (FTND; $n=280$ ).

\begin{tabular}{cccccc}
\hline Paso 1 & b & Wald & p & OR & 1,05 \\
\hline BDI & $-0,05$ & 6,602 & 0,01 & $1,01-1,09$ \\
Paso 2 & & & & 0,023 & 1,05 \\
\hline BDI & $-0,048$ & 5,145 & 0,399 & 0,80 \\
sexo & 0,226 & 0,711 & 0,009 & $1,01-1,09$ \\
FND $^{2}$ & $-0,140$ & 6,798 & $0,47-1,35$ & 1,15 \\
\hline
\end{tabular}

${ }^{1} \mathrm{BDI}=$ Inventario de Depresión de Beck.

${ }^{2} \mathrm{FTND}=$ Fagerström Test for Nicotine Dependence

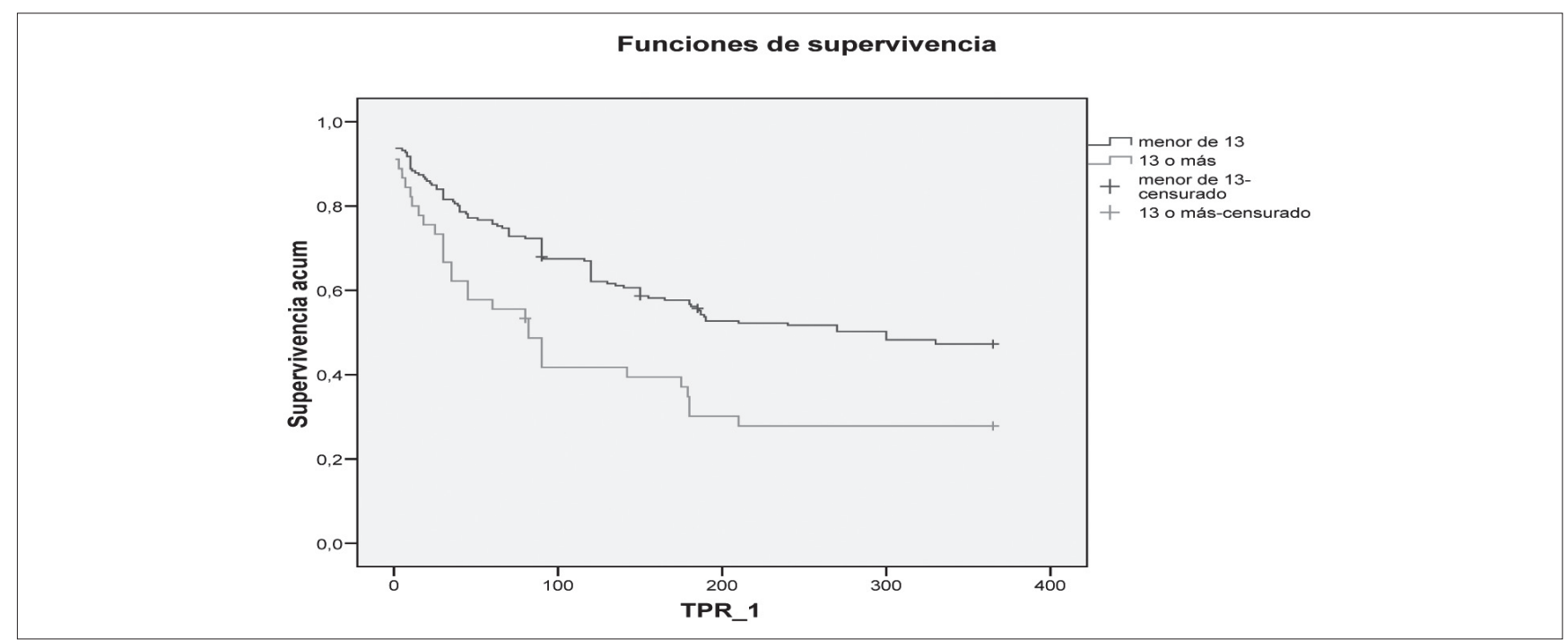

Figura 2. Curva de supervivencia utilizando modelo de Kaplan-Meier, según puntuaciones en $B D I<0 \geq 13$ al inicio de tratamiento y los días hasta la primera recaída. 


\section{Análisis de los antecedentes de TDM}

Durante la entrevista inicial, 41 (14,6\%) pacientes afirmaron tener antecedentes de depresión y haber necesitado tratamiento psiquiátrico o psicológico. Por sexos, los porcentajes fueron del 8,8\% para los hombres y el 22,4\% para las mujeres. Esta diferencia era estadísticamente significativa $\left(\nabla^{2}=8,81, g \mid=1, p=0,003\right)$. La presencia de antecedentes de depresión no se relacionó con la abstinencia en ningún momento de la evaluación.

\section{Análisis multivariable}

En el contexto de una regresión logística se introdujeron las covaribles sexo y FTND para estudiar su efecto en la relación sintomatología depresiva y abstinencia a los 3 meses. Se encontró que el sexo era independiente de dicha relación, y aunque la puntuación en el FTND se relacionó negativamente con la abstinencia, la relación entre sintomatología depresiva y abstinencia a los 3 meses era independiente del efecto de la severidad de la dependencia ya que el coeficiente de regresión era prácticamente el mismo con o sin la covariable FTND (-0,048 vs. $-0,05$; tabla 3$)$. Por otro lado, la toma de antidepresivos al inicio del tratamiento era igualmente independiente de la relación encontrada entre BDI y abstinencia a los 3 meses $(p=0,365)$.

\section{DISCUSIÓN}

La presencia de intensa sintomatología depresiva en el inicio del tratamiento para la deshabituación tabáquica es un factor de riesgo de recaída a corto, medio y largo plazo, cuando se desarrolla en el contexto laboral, independientemente del sexo, la severidad de la dependencia nicotínica y del tratamiento con antidepresivos. En segundo lugar, tener o no antecedentes de TDM es independiente del éxito del programa de cesación tabáquica para trabajadores. Todo ello confirma las hipótesis formuladas por los autores. Llama la atención, que la sintomatología depresiva no es una barrera para iniciar el tratamiento de deshabituación tabáquica, por lo que sujetos con ánimo depresivo (con mayor prevalencia en mujeres) participan habitualmente en intervenciones en el ámbito laboral. El tratamiento con bupropion no se relaciona con mayor probabilidad de abstinencia frente a otros fármacos independientemente del grado de sintomatología depresiva inicial. La toma de otros antidepresivos tampoco aumenta la probabilidad de abstinencia.

Este trabajo aborda una temática importante para la salud pública como es la identificación de barreras que impiden mejores resultados de programas de tratamiento de tabaquismo. Según la información disponible, éste es el primer estudio que evalúa el impacto de la sintomatología depresiva y los antecedentes de TDM en la abstinencia tabáquica, en el contexto de una intervención laboral. Hasta ahora, los estudios que han investigado posibles variables predictoras de abstinencia tabáquica en contextos laborales, no han evaluado la depresión ${ }^{27,28}$ o no han analizado su impacto en la abstinencia ${ }^{29}$. Los meta-análisis realizados sobre la eficacia de las intervenciones laborales tampoco refieren la depresión como una variable moduladora ${ }^{2,30}$. Además, la presente investigación tiene una elevada validez externa, puesto que se trata de una muestra amplia de trabajadores con distintas ocupaciones, que acuden voluntariamente, sin ser seleccionados por criterios de dependencia o de patología psiquiátrica y a los que no se asigna ningún tratamiento determinado.

Existen algunas limitaciones del presente estudio a considerar. El estudio analiza la presencia de sintomatología depresiva, no de la presencia de TDM, por ello es más difícil de generalizar los resultados a una población con entidad nosológica. Dicha sintomatología fue evaluada solo a nivel basal, por lo que se desconoce si aumentó o disminuyó durante el tratamiento y el efecto que ello pudo tener sobre la abstinencia. También debe tenerse en cuenta que los antecedentes de TDM fueron evaluados en una entrevista clínica en la que se pedía al sujeto dicha información, por ello, los TDM no diagnosticados no fueron detectados, por lo que puede haber una infra-representación de antecedentes de TDM. Se excluyeron casos de trastornos adaptativos, por lo que nuevamente los antecedentes de depresión podrian estar calculados con números menores a los reales. Ello puede haber influido tanto en la menor prevalencia de antecedentes de TDM encontrada en nuestra muestra comparada a la de otros estudios ${ }^{7}$, así como en la potencia estadística del análisis. De otro lado, la medición de la abstinencia mediante cooximetría se realizó en las visitas verificando la autodeclaración del sujeto en la abstinencia puntual. La abstinencia continuada fue reportada por el propio sujeto y no pudo ser objetivada más que en el momento de las visitas. A pesar de ello, este procedimiento (que incluye ambas medidas de abstinencia y la verificación mediante cooximetría en diferentes momentos del proceso, incluido los 12 meses) es el recomendado por el Subcomité de Verificación Bioquímica de la Society for Research on Nicotine and Tobacco ${ }^{31}$. Por último, la variable motivación pudo haber influido tanto en la asistencia voluntaria como en los resultados de abstinencia.

En cuanto al efecto de la sintomatología depresiva al inicio del tratamiento, los resultados coinciden con los hallazgos de otros estudios realizados en contextos no laborales ${ }^{19}$, siendo el efecto independiente de la presencia de historia de TDM contrariamente a lo que algunos estudios habian apuntado ${ }^{16}$. En cuanto al efecto de los antecedentes de TDM, los resultados discrepan de los resultados publicados por algunos estudios previos $s^{8,9}, 10,11$ pero son coherentes con las revisiones más recientes ${ }^{18}$, y una vez más se confirma la coincidencia con resultados de estudios realizados fuera del contexto laboral.

Teniendo en cuenta la relevancia del grado de sintomatología depresiva al inicio del tratamiento de deshabituación tabáquica en el contexto de las intervenciones laborales, nuevas investigaciones deberian corroborar nuestros hallazgos así como evaluar si la incorporación de otras estrategias terapéuticas, tanto psicológicas como farmacológicas, pueden aumentar la probabilidad de abstinencia en dichos sujetos. El tratamiento cognitivo-conductual con estrategias 
específicas para el manejo de los estados de ánimo mostró ser beneficioso para la población con depresión recurrente ${ }^{32,33}$, pero falló en mejorar los resultados en población con elevada sintomatología depresiva ${ }^{34}$. Es necesario pues, seguir investigando con el objetivo de aumentar las tasas de abstinencia en esta población.

\section{REFERENCIAS}

1. Encuesta Nacional de Salud 2006. Instituto Nacional de Estadistica. Disponible en: http://www.msc.es/estadEstudios/ estadisticas/encuestaNacional/encuesta2006.htm.

2. Moher M, Hey K, Lancaster T. Intervenciones en el lugar de trabajo para el abandono del hábito de fumar (Revisión Cochrane traducida). En: La Biblioteca Cochrane Plus, Número 2. Oxford: Update Software; 2006.

3. National Institute for Health and Clinical Experience. Workplace health promotion: how to help employees to stop smoking. London: National Institute for Health and Clinical Experience; 2007.

4. Kenney BA, Holahan CJ, North RJ, Holahan CK. Depressive symptoms and cigarette smoking in American workers. Am J Health Promot 2006; 20:179-82.

5. Anda RF, Williamson DF, Escobedo LG, Mast EE, Giovino GA, Remington PL. Depression and the dynamics of smoking. A national perspective. JAMA 1990; 264:1541-5.

6. Covey LS, Tam D. Depressive mood, the single-parent home, and adolescent cigarette smoking. Am J Public Health 1990; 80: 1330-3.

7. Wilhelm K, Wedgwood L, Niven H, Kay-Lambkin F. Smoking cessation and depression: current knowledge and future directions. Drug Alcohol Rev 2006; 25: 97-107.

8. Glassman AH, Stetner F, Walsh BT, Raciman PS, Fleiss JL, Cooper $T B$, et al. Heavy smokers, smoking cessation, and clonidine: Results of a double-blind, randomised trial. JAMA 1988; 259: 2863-6.

9. Glassman AH, Helzer JE, Covey LS, Cottler LB, Stetner F, Tipp JE, et al. Smoking, smoking cessation and major depression. JAMA 1990; 264: 1546-9.

10. Covey LS, Glassman AH, Stetner F, Becker J. Effect of history of alcoholism or major depression on smoking cessation. Am J Psychiatry 1993; 150: 1546-7.

11. Hall SM, Reus VI, Muñoz RF, Sees KL, Humfleet G, Hartz DT, et al. Nortriptyline and cognitive-behavioral therapy in the treatment of cigarette smoking. Arch Gen Psychiatry 1998; 55: 683-90.

12. Covey $L S$, Glassman $A H$, Stetner F. Depression and depressive symptoms in smoking cessation. Compr Psychiatry 1990; 31: 350-4.

13. Breslau N, Peterson EL, Schultz LR, Chilcoat HD, Andreski P. Major depression and stages of smoking. Arch Gen Psychiatry 1998; 55: 161-6.

14. Cox LS, Patten CA, Niaura RS, Decker AP, Rigotti N, Sachs DP, et al. Efficacy of bupropion for relapse prevention in smokers with and without a past history of major depression. J Gen Intern Med 2004; 19: 828-34.

15. Ginsberg JP, Klesges RC, Johnson KC, Eck LH, Meyers AW, Winders $\mathrm{SA}$. The relationship between a history of depression and adherence to a multicomponent smoking-cessation program. Addict Behav 1997; 22: 783-7.

16. Hall S, Muñoz R, Reus V. Cognitive-behavioral intervention increases abstinence rates for depressive-history smokers. J Consult Clin Psychol 1994; 62: 141-6.
17. Hayford KE, Patten $C A$, Rummans $T A$, Schroeder DR, Offord KP, Croghan IT, et al. Efficacy of bupropion for smoking cessation in smokers with a former history of major depression or alcoholism. Br J Psychiatry 1999; 174: 173-8.

18. Hitsman B, Borrelli B, McChargue DE, Spring B, Niaura R. History of depression and smoking cessation outcome: a meta-analysis. J Consult Clin Psychol 2003; 71: 657-63.

19. Kinnunen T, Doherty K, Militello FS, Garvey AJ. Depression and smoking cessation: characteristics of depressed smokers and effects on nicotine replacement. J Consult Clin Psychol 1996; 64: 791-8.

20. Hughes JR, Stead LF, Lancaster T. Antidepressants for smoking cessation. In: Cochrane Database of Systematic Reviews, Issue 4. Oxford: Update Software; 2006.

21. Fiore MC, Jaén CR, Baker TB, Bailey WC, Benowitz NL, Curry SJ, et al. Treating Tobacco Use and Dependence: 2008 Update. Clinical practice guideline. Rockville: U.S. Department of Health and Human Services, Public Health Service; 2008.

22. Conde V, Useros E. Adaptación castellana de la escala de evaluación conductual para la depresión de Beck. Rev Psiquiatr Psicol Med Eur Am Lat 1975; 12: 217-36.

23. Heatherton TF, Kozowski LT, Frecker RC, Fagerström KO. The Fagerström test for nicotine dependence: a revision of the Fagerström Tolerance Questionnaire. Brit J Addict 1991; 86:1119-27.

24. Cummings $S R$, Richard RJ. Optimum cutoff points for biochemical validation of smoking status. Am J Public Health 1988; 78, 574-5.

25. Hughes JR, Keely JP, Niara RS, Ossip-Klein DJ, Richmond RL, Swan Ge. Measures of abstinence in clinical trials: issues and recommendations. Nicotine Tob Res 2003; 5:13-25.

26. Michalak EE, Murria G, Wilkinson $C$, Dowrick $C$, Lasa L, Lehtinen $V$, et al. Estimating depression prevalence from the Beck Depression Inventory: is season of administration a moderator? Psychiatry Res 2004;129: 99-106.

27. Wetter DW, McClure JB, de Moor C, Cofta-Gunn L, Cummings $S$, Cinciripini PM, et al. Concomitant use of cigarettes and smokeless tobacco: prevalence, correlates, and predictors of tobacco cessation. Prev Med 2002; 34: 638-48.

28. Razavi $D$, Vandecasteele $H$, Primo $C$, Bodo $M$, Debrier $F$, Verbist $\mathrm{H}_{\text {, et }}$ al. Maintaining abstinence from cigarette smoking: effectiveness of group counselling and factors predicting outcome. Eur J Cancer 1999; 35: 1238-47.

29. Lang $T$, Nicaud V, Slama K, Hirch A, Imbernon E, Goldberg $M$, et al. Smoking cessation at the workplace. Results of a randomised controlled intervention study. J Epidemiol Community Health 2000; 54: 349-54.

30. Smedslund G, Fisher KJ, Boles SM, Lichtenstein E. The effectiveness of workplace smoking cessation programmes: a meta-analysis of recent studies. Tob Control 2004;13:197-204.

31. Society for Research on Nicotine and Tobacco Subcommittee on Biochemical Verification. Biochemical verification of tobacco use and cessation. Nicotine Tob Res 2002; 4, 149-59.

32. Brown RA, Kahler CW, Niaura R, Abrams DB, Sales SD, Ramsey SE, et al. Cognitive-behavioral treatment for depression in smoking cessation. J Consult Clin Psychol 2001; 69: 471-80.

33. Hass AL, Muñoz RF, Humfleet GL, Reus V, Hall SM. Influences of mood, depression history, and treatment modality on outcomes in smoking cessation. J Consult Clin Psychol 2004; 72: 563-70.

34. Brown RA, Niaura R, Lloyd-Richardson EE, Strong DR, Kahler $\mathrm{CW}$, Abrantes AM, et al. Bupropion and cognitive-behavioral treatment for depression in smoking cessation. Nicotine Tob Res 2007; 9: 721-30. 